\title{
Primary pulmonary myxoid sarcoma with EWSR1-CREB1 fusion: a case report and review of the literature
}

\author{
Zhenwei Chen ${ }^{1}$, Yihui Yang ${ }^{1}$, Rongming Chen ${ }^{2}$, Chi Sing $\mathrm{Ng}^{3^{*}}$ (D) and Hongqi Shi ${ }^{1}$
}

\begin{abstract}
Background: Primary pulmonary myxoid sarcoma (PPMS) is an extremely rare lung sarcoma that is characterized in most cases by recurrent balanced chromosomal translocation $\mathrm{t}(2 ; 22)(\mathrm{q} 33 ; \mathrm{q} 12)$ leading to the oncogenic fusion gene EWSR1-CREB1.

Case presentation: We report a case of PPMS with molecular confirmation using fluorescence in situ hybridization (FISH) and DNA sequencing in a 45-year-old female patient. Computer tomography (CT) scanning revealed a peripheral circumscribed solid mass of $2.1 \times 2 \mathrm{~cm}$ in the right lung superior lobe. Histologically, the tumor cells ranged from stellate, polygonal to chondrocyte-like or physaliferous-like, forming reticular network of delicate lacelike cellular strands and cords in abundant myxoid stroma. The tumor cell immunophenotype was positive for vimentin, EMA and negative for CK-pan, TTF-1, CAM5.2, S-100, calponin, SMA, desmin, ALK, CD31 and CD34. Molecular analysis demonstrated EWSR1-CREB1 gene fusion in this tumor. During 38 months of follow-up, the patient was alive with no clinical or radiological evidence of recurrence or metastasis.

Conclusion: PPMS is a rare low-grade sarcoma with distinct histological and genetic features. We add another case to the literature of this rare tumor and report for the first time occurrence of chondrocyte-like and physaliferous-like tumor cells in this tumor, thus enriching its morphologic and cytologic spectrum.
\end{abstract}

Keywords: Pulmonary, Myxoid, Sarcoma, Chondrocyte-like, Physaliferous-like, EWSR1, CREB1

\section{Background}

Primary pulmonary sarcomas are extremely rare with prevalence of about $0.2 \%$ [1]. They comprise a heterogeneous group of sarcomas morphologically similar to the soft tissue counterparts. Among these rare sarcomas, primary pulmonary myxoid sarcoma (PPMS) are even more infrequent. PPMS is a recently described lung sarcoma more prevalent in young females with a characteristic genetic EWSR1-CREB1 fusion in most cases [2,3].. To the best of our knowledge, 25 cases have been reported in the English literature [2-11], among which only 17 cases were confirmed with presence of the EWSR1-CREB1 gene fusion. We report another case of PPMS harboring the EWSR1-CREB1 gene fusion confirmed by molecular method, with review of the literature.

\footnotetext{
* Correspondence: ngcspeter@gmail.com

${ }^{3}$ Department of Pathology, St. Teresa's Hospital, Kowloon, Hong Kong

Full list of author information is available at the end of the article
}

\section{Case presentation}

A 45-year-old woman was referred to our hospital for physical checkup. She was asymptomatic with no pulmonary obstructive symptoms or pneumonia. The patient was a non-user of alcohol and tobacco products. General physical and laboratory examination was unremarkable. Chest computed tomography (CT) revealed a $2.1 \times 2 \mathrm{~cm}$ peripheral solid mass in the right lung superior lobe featuring moderate heterogeneous enhancement (Fig. 1). There was no abnormality on bronchoscopic and cytological examinations. Routine blood tests and tumor marker levels were normal. Although CT findings suggested the possibility of hemangioma, the patient insisted on surgical excision. During surgery, a mass was detected in the right lung superior lobe which adhered to the superior vena cava with no invasion to the adjacent lung parenchyma or bronchus. Adjuvant chemotherapy was not given post-operatively. At follow-up 38

(c) The Author(s). 2020 Open Access This article is distributed under the terms of the Creative Commons Attribution 4.0 International License (http://creativecommons.org/licenses/by/4.0/), which permits unrestricted use, distribution, and 


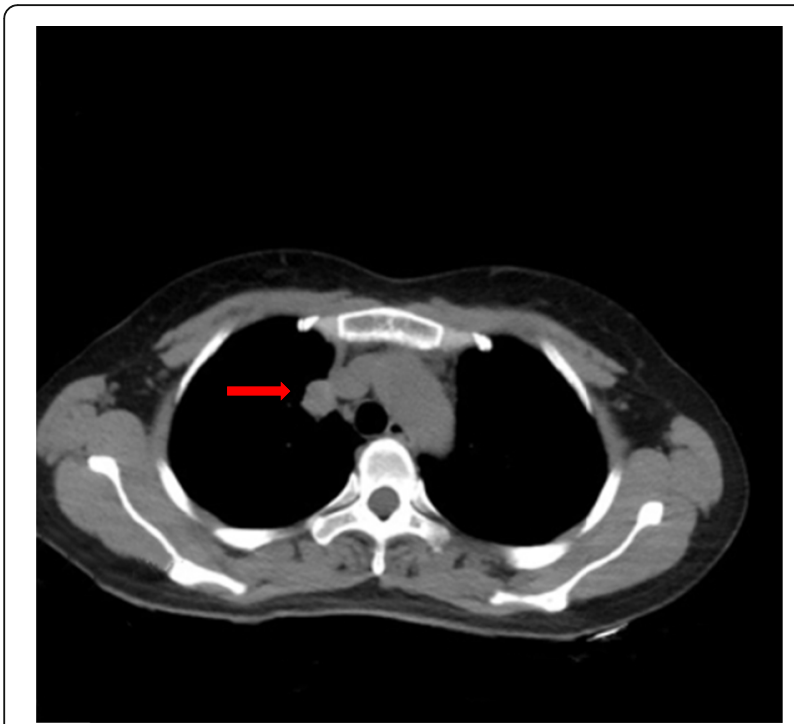

Fig. 1 Chest computed tomography showed a $2.1 \times 1.7 \mathrm{~cm}$ welldefined round mass exhibiting mild, heterogeneous internal enhancement at the periphery of the right superior lobe

months after surgery, there was no evidence of recurrence or metastasis.

Macroscopically, the tumor was a solitary, wellcircumscribed mass with a fleshy homogeneous white and gelatinous cut surface, measuring $2 \times 1.7 \times 1 \mathrm{~cm}$ without invasion to the bronchus. The tumor was histologically multinodular and composed of oval, or polygonal cells with vesicular nuclei, reminiscent of chondrocyte-like or physaliferous-like cells, in a background of myxoid stroma (Fig. 2a-d). There were rare mitotic figures and rich lymphoplasmacytic cell infiltration was also evident (Fig. 2e). There was no evidence an endobronchial component. Immunophenotypically, the tumor was positive for vimentin (Fig. 2f), epithelial membrane antigen (EMA) (Fig. 2g) and negative for CKpan, CAM5.2, CD31, CD34, smooth muscle actin (SMA), desmin (Fig. 2h), anaplastic lymphoma kinase (ALK) (Fig. 2i), calponin, TTF-1 and S-100 protein (Fig. 2j) (Table 1). Molecular analysis was performed on formalin fixed paraffin embedded (FFPE) material by fluorescence in situ hybridization (FISH) using LSI EWSR1 dual-color break-apart probe (Break Apart Rearrangement probe, Ambipin,China), and specific gene fusion transcripts by reverse transcription-polymerase chain reaction (RT-PCR). Primers for the RT-PCR were located in exon 7 of EWSR1 (5'TCCTACAGCCAAGCTCCAAGTC3') and in exon 7 of CREB1 (5'GTACCCCATCGGTACCATTGT3'). FISH showed a clear separation of red and green signals within a single tumor cell, demonstrating the presence of a EWSR1 gene rearrangement (Fig. 3a). The RT-PCR gene fusion products were confirmed by DNA sequencing with Sanger method (ABI3730, Japan) to show in-frame fusion of the $5^{\prime}$ region of EWSR1 (exon 7) to the 3' region of CREB1 (exon 7) (Fig. 3b \& c). The histological, immunophenotypic and molecular findings confirmed the diagnosis of primary pulmonary myxoid sarcoma with EWSR1$C R E B 1$ gene fusion.

\section{Discussion}

Nicholson et al. first described a rare primary pulmonary myxoid sarcoma in 1999 as a novel low-grade malignant myxoid endobronchial neoplasm [4]. Further studies showed that this tumor has a characteristic genetic fingerprint characterized by the oncogenic fusion gene EWSR1CREB1 [2], which has been first included in the latest WHO fascicle as a characteristic of this tumor [12]. To date, 25 cases of this sarcoma have been described in the English literature [2-11],with only 17 confirmed by presence of EWSR1-CREB1 fusion [2, 3, 5-10]. Our case is the eighteenth case of PPMS with confirmed EWSR1-CREB1 gene fusion. All 26 cases (including the 5 cases with no EWSR1-CREB1 gene fusion, 1 case with ESWR1 rearrangement not involving $C R E B 1$ and 2 cases without molecular genetic workup) are reviewed and summarized in Table 2. The patients had a broad age range from 21 to 80 years (mean 43 years), with female predominance (female: male, 1.5:1). The clinical manifestations are relatively nonspecific and include cough, hemoptysis and weight loss. There was no definite site of predilection of this tumor in the lung. Although initially reported mostly as endobronchial growths related to the bronchial tree, there were some tumors not associated with the bronchus $[7,8,10]$, as in our case. One such case was a EWSR1$C R E B 1$ gene rearranged low grade myxoid sarcoma of the pulmonary artery [10]. Grossly, the excised lesions are well circumscribed, ranged in size from 1.5 to $14 \mathrm{~cm}$ (average $4 \mathrm{~cm}$ ) with pale, glistening cut surface. Microscopically, the characteristic features of most cases are lobulated architecture with cords of ovoid, spindle or stellate cells embedded in a prominent myxoid matrix. Most cases have a patchy background of inflammatory cells consisting mainly of lymphocytes and plasma cells. In distinct contrast, the tumor cells in our case are chondrocyte-like or physaliferous-like. Predominance of chondrocyte-like and physaliferous-like tumor cells in PPMS has not been reported in the literature previously. The inflammatory infiltrate in our case is also more intense. These unique findings of our case enrich the morphologic and cytologic spectrum of PPMS. Regarding genetics of PPMS, genetic study was not performed in two of the 26 cases. Among the remaining 24 cases, 18 (including our case) showed EWSR1-CREB1 gene fusion, one showed EWSR1 rearrangement but not fusion with CREB1 (case 16) [6], and 5 were negative for ESWR1-CREB1 gene fusion. This 

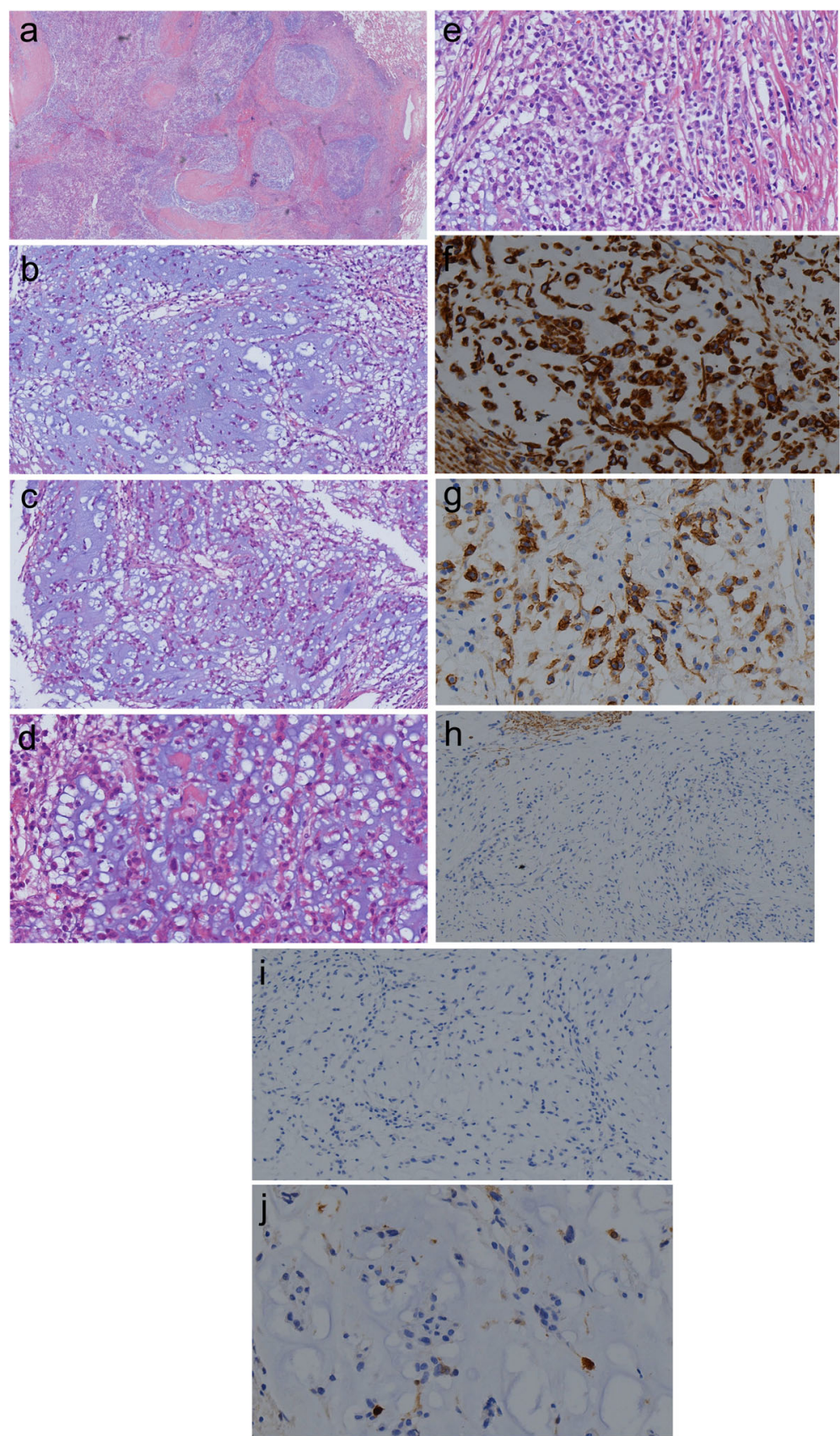

Fig. 2 a. The tumor had abundant myxoid stroma and a multinodular architecture at low-magnification (a: magnification $\times 20$ and $\mathbf{b}$ magnification $\times 100$ ). b \& c \& d The tumor showed variable cellularity with polygonal, stellate to chondrocyte-like or physaliferous-like tumor cells organized in prominent reticular network of delicate lace-like cellular strands and cords within prominent myxoid stroma (b \& $\mathbf{c}$ magnification $\times$ 100, d: magnification $\times 400$ ). e. Abundant lymphoid cells and plasma cells at the periphery or within the tumor (magnification $\times 400) \mathbf{f}$. Tumor cell immunohistochemical positive expression of vimentin (magnification $\times 400$ ). $\mathbf{g}$. Tumor cell immunohistochemical positive expression of EMA (magnification $\times 400$ ). $\mathbf{h}$. Tumor cell immunohistochemical negative expression of Desmin (magnification $\times 200$ ). i. Tumor cell immunohistochemical negative expression of ALK (magnification $\times 400$ ). j. Tumor cell immunohistochemical negative expression of S-100 (magnification $\times 400)$

makes a $75 \%$ positive rate for EWSR1-CREB1 fusion, 79\% for EWSR1 rearrangement, and a negative rate of $21 \%$ for ESWR1-CREB1 fusion or ESWR1 rearrangement in the 24 cases where genetic study was performed in the reviewed series of PPMS. These findings are broadly similar to the previously reported EWSR1-CREB1 gene fusion rate of $70 \%$ [2] and 63\% [3], and EWSR1 and/or CREB1 gene rearrangement rate of $89 \%$ [3] in two smaller series of 
Table 1 List of antibodies

\begin{tabular}{llll}
\hline Antigen & Clone & Dilution & Manufacturer \\
\hline Vimentin & MX034 & $1: 200$ & Fuzhou MXB Biotechnology Co,Ltd. China \\
EMA & GP1.4 & $1: 100$ & Fuzhou MXB Biotechnology Co,Ltd. China \\
CK-pan & polyclonal & $1: 200$ & Fuzhou MXB Biotechnology Co,Ltd. China \\
CAM5.2 & CAM5.2 & $1: 100$ & Fuzhou MXB Biotechnology Co,Ltd. China \\
CD31 & JC/70A & $1: 100$ & Fuzhou MXB Biotechnology Co,Ltd. China \\
CD34 & QBEnd/10 & $1: 100$ & Fuzhou MXB Biotechnology Co,Ltd. China \\
SMA & IA4 & $1: 200$ & Fuzhou MXB Biotechnology Co,Ltd. China \\
Calponin & MX023 & $1: 300$ & Fuzhou MXB Biotechnology Co,Ltd. China \\
TTF-1 & 8G7G3/1 & $1: 200$ & Fuzhou MXB Biotechnology Co,Ltd. China \\
S-100 & polyclonal & Fuzhou MXB Biotechnology Co,Ltd. China \\
Desmin & D33 & $1: 200$ & Guangzhou Onco Care Biotechnology Co,Ltd. China \\
ALK & MX064 & $1: 200$ & Guangzhou Onco Care Biotechnology Co,Ltd. China \\
\hline
\end{tabular}

PPMS. Thway $\mathrm{K}$ et al [2] detected EWSR1-CREB1 fusion gene in 7 PPMS, with the fusion loci located in exon 7 of EWSR1 and exon 7 of CREB1 in 6 of the 7 cases. One other case showed fusion loci in exon 7 of EWSR1 and exon 8 of CREB1. There was no histological difference in the tumors bearing the different gene fusions. Furthermore, one case in this series with no EWSR1-CREB1 fusion died from brain metastasis, while another case bearing the EWSR1-CREB1 fusion of the same series had kidney metastasis but was alive and well after 3 years [2]. In another series [7], one case with EWSR1-CREB1 fusion developed metastasis to the contralateral lung, but remained disease free 72 months after removal of the lung metastasis. It appears that PPMS with EWSR1-CREB1 fusion fares better than those without EWSR1-CREB1 fusion. However, whether presence of EWSR1-CREB1 fusion connotates better prognosis in PPMS requires further study.

PPMS should be differentiated from extraskeletal myxoid chondrosarcoma (EMC), which can also arise in the lung $[13,14]$. Histologically, EMC is composed of cords of cells with scarce cytoplasm immersed in abundant myxoid matrix similar to PPMS. EMC, however, at least focally expresses S-100 which is negative in PPMS, as in our case. Genetically, EMC may harbor EWSR1NR4A3、TAF15-NR4A3 or TFG-NR4A3 gene fusion [15, 16], and may thus confound with PPMS. However, PPMS exhibits the characteristic EWSR1-CREB1 fusion gene, allowing distinction from EMC. Differentiation between PPMS and angiomatoid fibrous histiocytoma $(\mathrm{AFH})$, which may occur in the lung [17], is more problematic. Although both tumors are located predominantly endobronchially and harbor the EWSR1-CREB1 fusion gene, they differ in morphology. PPMS is composed of cords and clusters of spindle, stellate, ovoid, and in our case chondrocyte-like and physaliferous-like tumor cells arranged in a reticular pattern within prominent alcian blue positive myxoid stroma. In contrast, AFH comprises sheets and islands of spindle to epithelioid cells with bland ovoid vesicular nuclei and abundant eosinophilic cytoplasm within loose stroma. PPMS has more abundant myxoid stroma and lacks the prominent peripheral cuff of lymphocytes usually present in AFH. Moreover, desmin is expressed in 50\% of $\mathrm{AFH}$, which is not present in PPMS. Furthermore, ALK expression is common in AFH, which is negative in PPMS. Finally, AFH may show other fusion genes, such as EWSR1-ATF1 and FUS-ATF1, both of which are absent in PPMS. PPMS should be also be distinguished from myoepithelial tumors, which can also arise in the lung [18] with endobronchial growth pattern and EWSR1 rearrangements. Myoepithelial tumors are immunohistochemically positive for CK, p63, SMA, calponin and S-100 protein, which are negative in PPMS. Pulmonary microcystic fibromyxoma (PMF) are composed of bland spindle to stellate cells with uniform nuclei widely spaced within fibromyxoid stroma which is alcian blue positive and hyaluronidase sensitive [19]. However, PMF are not endobronchially located and are much less cellular with bland stellate cells disposed in a microcystic pattern. Furthermore, PMF does not harbor the EWSR1-CREB1 gene fusion, distinct from PPMS. Another differential diagnosis is inflammatory myofibroblastic tumor (IMT) which may possess myxoid stroma and inflammatory infiltrates. The stellate tumor cells in IMT are immunophenotypically positive for SMA, desmin and ALK, which are negative in PPMS. Since the physaliferous-like tumor cells in PPMS exhibit cytoplasmic bubbles, they can potentially be confused with lipoblasts. PPMS thus needs to be differentiated from low grade primary or metastatic myxoid liposarcoma [20]. Myxoid liposarcoma, however, often harbors rearrangement involving the DDIT3 gene, which is not present in PPMS. The various differential diagnoses and their features are summarized in Table 3. 

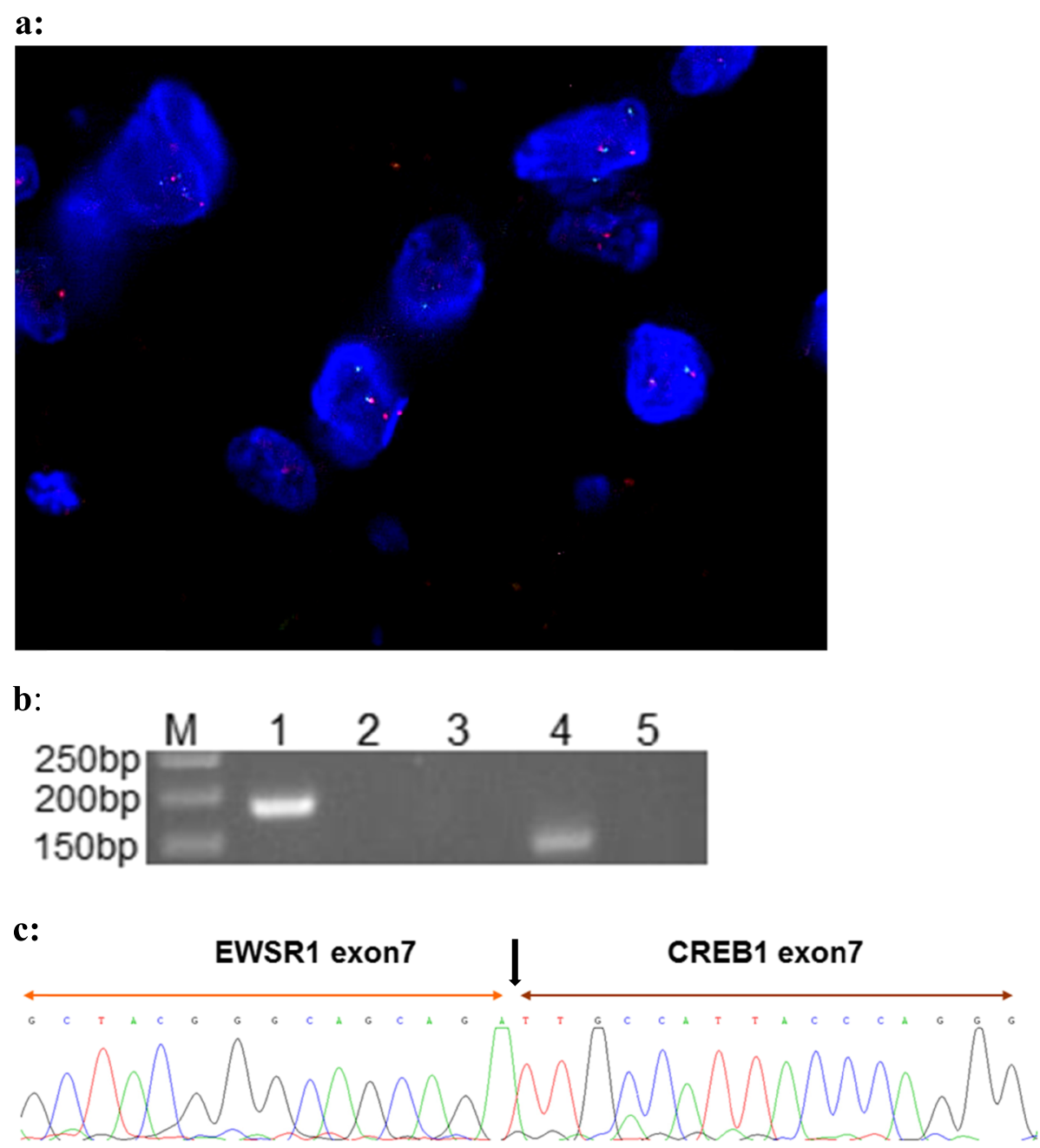

Fig. 3 a: Dual color interphase fluorescence in situ hybridization utilizing the EWSR1 break-apart probe. Split red and green signals within a single tumor cell demonstrated the presence of EWSR1 rearrangement. $\mathbf{b}$ : Gel electrophoresis of the RT-PCR products using EWSR1 and CREB1 primers; confirming presence of ESWR1-CREB1 fusion in the patient's sample (Lane 4). M:50 bp markers; Lane 1: Internal control, PGK; Lane 2: Negative control with EWSR1 exon $7+$ CREB1 exon 7 fusion primer; Lane 3: Negative control with EWSR1 exon $7+C R E B 1$ exon 8 fusion primer; Lane 4: Patient's sample with EWSR1 exon $7+$ CREB1 exon 7 fusion primer; Lane 5: Patient's sample with EWSR1 exon $7+C R E B 1$ exon 8 fusion primer. c: Sanger sequencing result of the patient's RT-PCR product demonstrated in Lane 4 of $\mathbf{b}$. The sequence was the same as the EWSR1 - CREB1 fusion gene as reported in the literature

Although most patients treated by adequate surgery had no local recurrences, three patients developed metastasis within a follow up period from 4 months to 15 years. The metastatic sites included the contralateral lung [7], the left kidney and brain [2]. The patient with brain metastasis died a few months after surgery. The patient with metastasis to the left kidney is still alive and well [2]. The patient with metastasis to the contralateral lung occurred 7 months after surgery [7]. Though PPMS is considered a low-grade sarcoma, it appears that there are no reliable histological or clinical features for predicting its prognosis and outcome. Some cases histologically showing nuclear atypia, necrosis and high mitotic figures, with increased Ki-67 proliferative index did not fare worse prognosis [2]. On the other hand, endobronchial location, capsule-like fibrosis, absence of solid architecture and lack of necrosis might be predictive of more favorable outcome, though presence of endobronchial component and capsule-like features had also been observed in metastatic cases [2]. Molecular fingerprint may play a prognostic role, as patients with EWSR1 rearrangement [11] or EWSR1-CREB1 gene fusion $[2,7]$ may have more favorable prognosis, while those with no EWSR1-CREB1 fusion or wild type EWSR1 may portend poor clinical outcome $[2,11]$.

\section{Conclusion}

PPMS is a rare low-grade sarcoma that occurs mostly in middle age women with most harboring the characteristic EWSR1-CREB1 fusion gene. Our case adds to the 


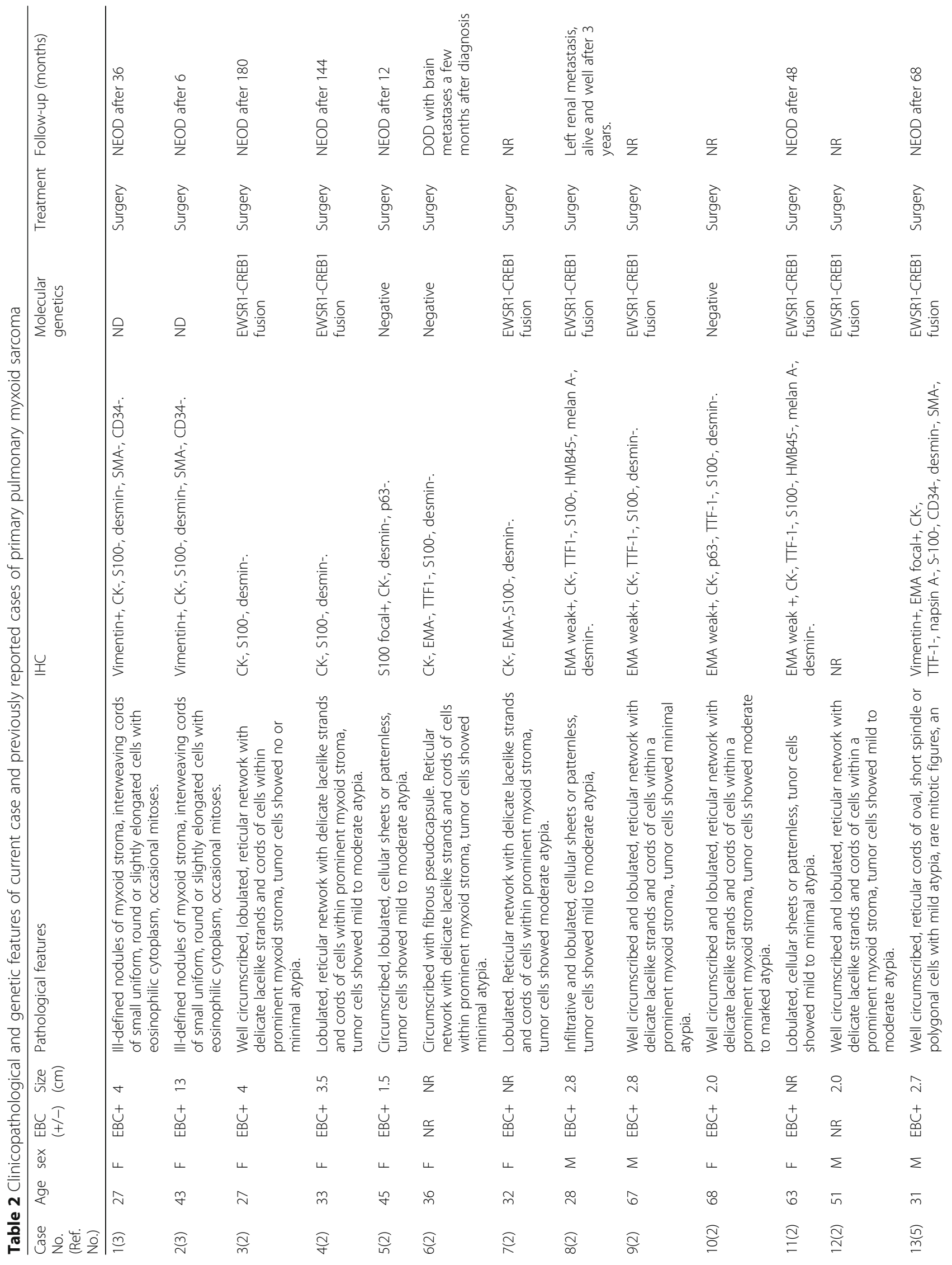




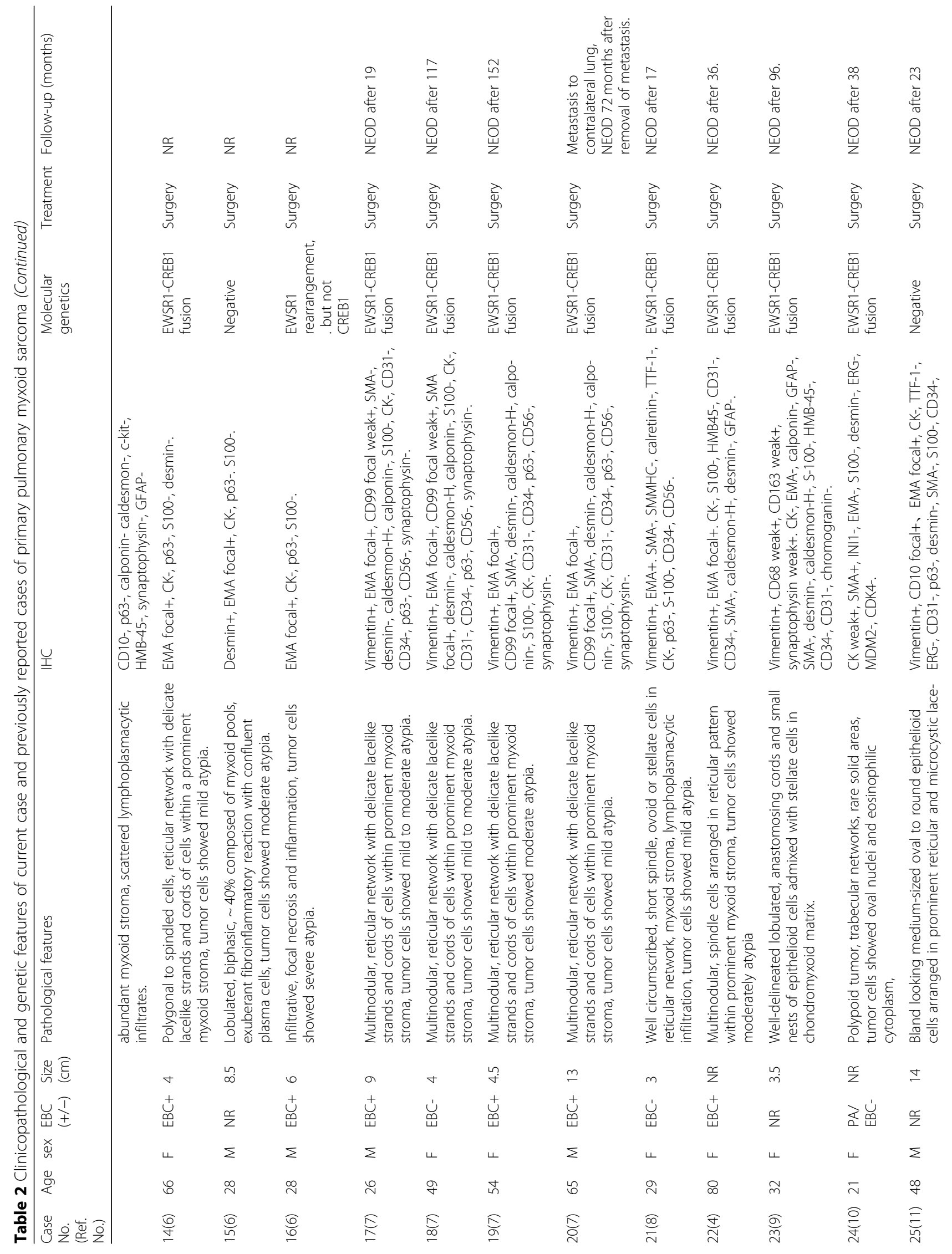




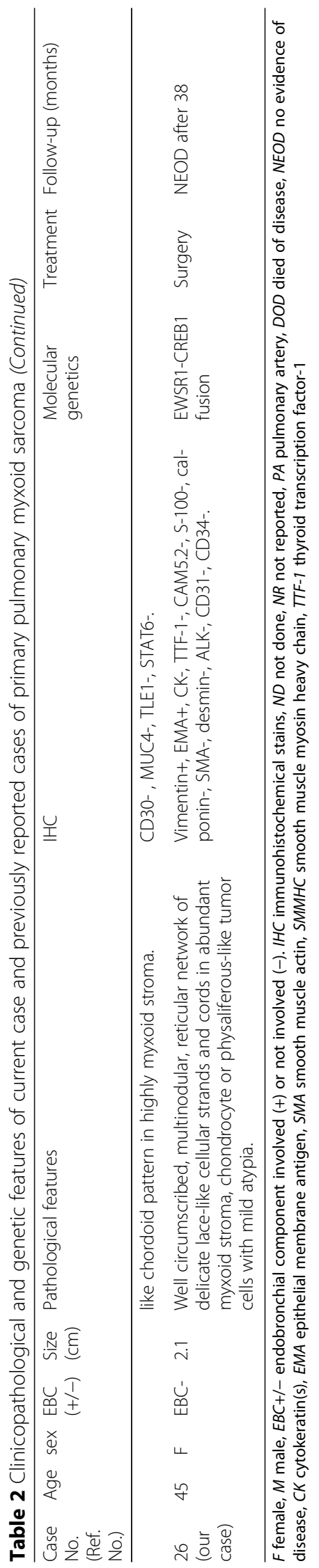




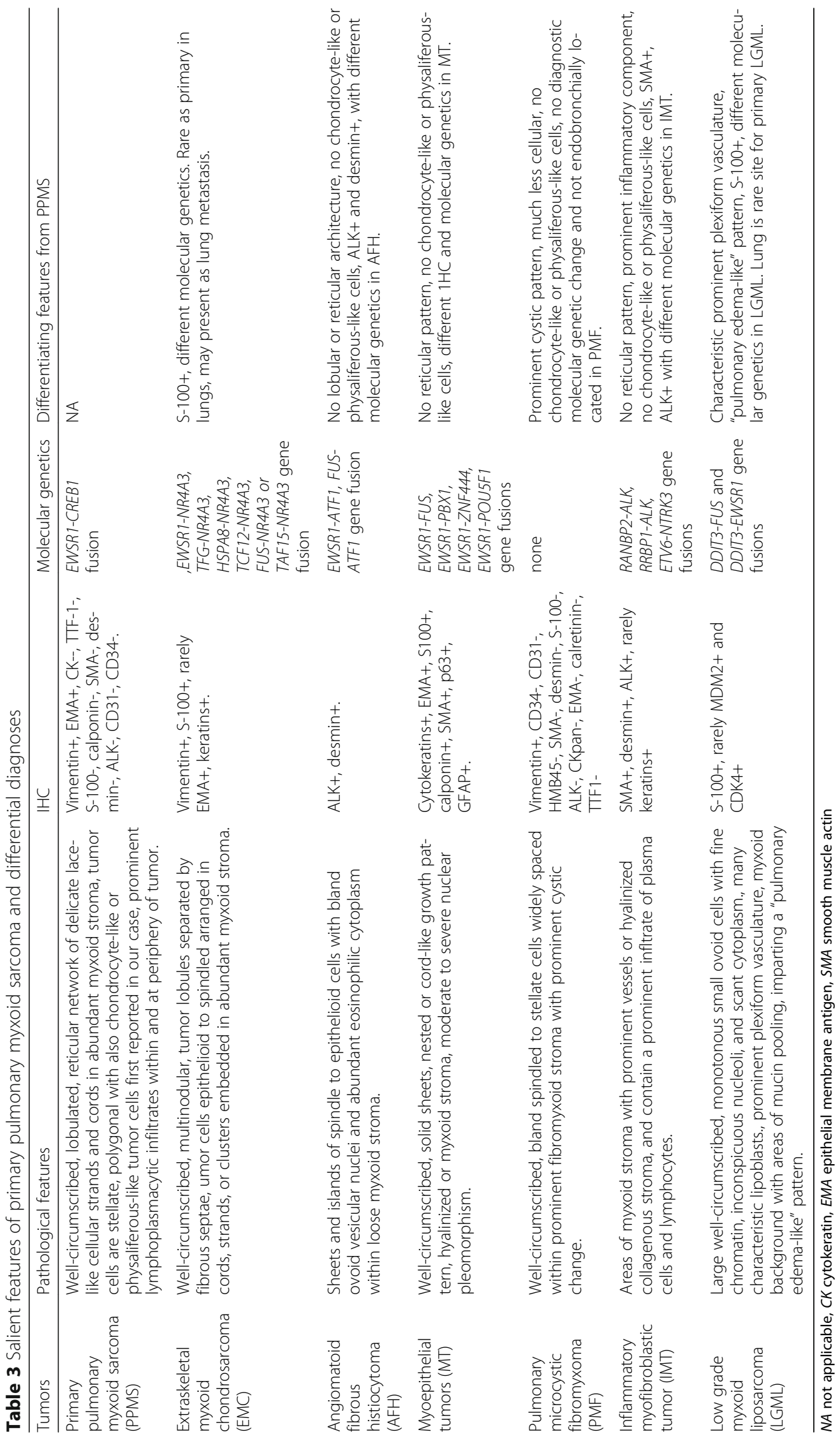


literature of this rare tumor and enriches its morphologic and cytologic spectrum with occurrence of chondrocyte-like and physaliferous-like tumor cells. Early diagnosis of PPMS requires high index of suspicion in pulmonary myxoid tumors and is based on clinical features, histological and molecular studies. The clinical behavior of this tumor is still uncertain due to its rarity and limited follow up of reported cases. There are no reliable histological or clinical features to predict prognosis and outcome of this tumor.

\section{Abbreviations}

AFH: Angiomatoid fibrous histiocytoma; ALK: Anaplastic lymphoma kinase; CAM5.2: Cytokeratin; CK-pan: Cytokeratin (Pan); CT: Computer tomography; EMA: Epithelial membrane antigen; EMC: Extraskeletal myxoid chondrosarcoma; FFPE: Formalin fixed and paraffin embedded; FISH: Fluorescence in situ hybridization; IMT: Inflammatory myofibroblastic tumor; PMF: Pulmonary microcystic fibromyxoma; PPMS: Primary pulmonary myxoid sarcoma; RT-PCR: Reverse transcription-polymerase chain reaction; SMA: Smooth muscle actin; TTF-1: Thyroid transcription factor 1

\section{Acknowledgements}

We thank Xianbin-tang and Yinghua-Hao, Department of Pathology, Taihe Hospital, Hubei University of Medicine, for their technical support (FISH and RT-P(R).

\section{Authors' contributions}

ZWC and RMC performed histological evaluation, made the pathological diagnosis and drafted the manuscript. YHY and HQS performed the immunohistochemistry. CSN advised on manuscript preparation and made revision to the final manuscript. All authors read and approved the final manuscript.

\section{Funding}

Nil.

\section{Availability of data and materials}

The dataset supporting the conclusion of this article is included within the article.

\section{Ethics approval and consent to participate}

Not applicable.

\section{Consent for publication}

Written informed consent was obtained from the patient for the publication of this case report and any accompanying images. A copy of the consent form is available for review by the Editor of Diagnostic Pathology.

\section{Competing interests}

The authors declare that they have no competing interests.

\section{Author details}

'Department of Pathology, Jinhua Municipal Central Hospital, 351 Mingyue Road, Jinhua 321000, Zhejiang Province, People's Republic of China. ${ }^{2}$ Department of Pathology, the People's Hospital of Changfeng County, Changfeng County, Anhui Province, People's Republic of China. ${ }^{3}$ Department of Pathology, St. Teresa's Hospital, Kowloon, Hong Kong.

Received: 18 August 2019 Accepted: 5 February 2020

Published online: 10 February 2020

\section{References}

1. Attanoos RL, Appleton MA, Gibbs AR. Primary sarcomas of the lung: a clinicopathological and immunohistochemical study of 14 cases. Histopathology. 1996;29:29-36.

2. Thway K, Nicholson AG, Lawson K, Gonzalez D, Rice A, Balzer B, et al. Primary pulmonary myxoid sarcoma with EWSR1-CREB1 fusion: a new tumor entity. Am J Surg Pathol. 2011;35:1722-32.
3. Prieto-Granada CN, Ganim RB, Zhang L, Antonescu C, Mueller J. Primary pulmonary myxoid sarcoma: A newly described entity-report of a case and review of the literature. Int J Surg Pathol. 2017;25:518-25.

4. Nicholson AG, Baandrup U, Florio R, Sheppard MN, Fisher C. Malignant myxoid endobronchial tumour: a report of two cases with a unique histological pattern. Histopathology. 1999;35:313-8.

5. Matsukuma S, Hisaoka M, Obara K, Kono T, Takeo H, Sato K, et al. Primary pulmonary myxoid sarcoma with EWSR1-CREB1 fusion, resembling extraskeletal myxoid chondrosarcoma: case report with a review of literature. Pathol Int. 2012;62:817-22.

6. Smith SC, Palanisamy N, Betz BL, Tomlins SA, Mehra R, Schmidt LA, et al. At the intersection of primary pulmonary myxoid sarcoma and pulmonary angiomatoid fibrous histiocytoma: observations from three new cases. Histopathology. 2014;65:144-6.

7. Jeon YK, Moon KC, Park SH, Chung DH. Primary pulmonary myxoid sarcomas with EWSR1-CREB1 translocation might originate from primitive peribronchial mesenchymal cells undergoing (myo) fibroblastic differentiation. Virchows Arch. 2014;465:453-61.

8. Kim S, Song SY, Yun JS, Choi YD, Na KJ. Primary pulmonary myxoid sarcoma located in interlobar fissure without parenchymal invasion. Thorac Cancer. 2017:8:535-8.

9. Yanagida R, Balzer BL, Mckenna RJ, Fuller CB. Primary pulmonary myxoid sarcoma, a potential mimic of metastatic extraskeletal myxoid chondrosarcoma. Pathology. 2017:49:792-4.

10. Opitz I, Lauk O, Schneiter D, Ulrich S, Maisano F, Weder W, et al. Intraluminal EWSR1-CREB1 gene rearranged, low-grade myxoid sarcoma of the pulmonary artery resembling extraskeletal myxoid chondrosarcoma. Histopathology. 2019;74:526-30.

11. Agaimy A, Duell T, Morresi-Hauf AT. EWSR1-fusion-negative, SMARCB1deficient primary pulmonary myxoid sarcoma. Pol J Pathol. 2017;68:261-7.

12. Travis WD, Brambilla E, Burke AP, Marx A, Nicholson AG. WHO Classification of Tumours of the Lung, Pleura, Thymus and Heart. Lyon: IAPR press; 2016. p. P129-31.

13. Balanzá R, Arrangoiz R, Cordera F, Muñoz M, Luque-de-León E, Moreno E, et al. Pulmonary extraskeletal myxoid chondrosarcoma: A case report and literature review. Int J Surg Case Rep. 2016;27:96-101.

14. Zhou Q, Lu G, Liu A, Kohno T. Extraskeletal myxoid chondrosarcoma in the lung: asymptomatic lung mass with severe anemia. Diagn Pathol. 2012;7: 112.

15. Thway K, Fisher C. Tumors with EWSR1-CREB1 and EWSR1-ATF1 fusions: the current status. Am J Surg Pathol. 2012;36:e1-e11.

16. Hisaoka M, Hashimoto H. Extraskeletal myxoid chondrosarcoma: updated clinicopathological and molecular genetic characteristics. Pathol Int. 2005;55: 453-63.

17. Thway K, Nicholson AG, Wallace WA, Al-Nafussi A, Pilling J, Fisher C. Endobronchial pulmonary angiomatoid fibrous histiocytoma: two cases with EWSR1-CREB1 and EWSR1-ATF1 fusions. Am J Surg Pathol. 2012;36:883-8.

18. Leduc C, Zhang L, Öz B, Luo J, Fukuoka J, Antonescu CR, et al. Thoracic myoepithelial tumors: a pathologic and molecular study of 8 cases with review of the literature. Am J Surg Pathol. 2016;40:212-23.

19. Shilo K, Miettinen M, Travis WD, Timens W, Nogueira R, Franks TJ. Pulmonary microcystic fibromyxoma: report of 3 cases. Am J Surg Pathol. 2006;30: 1432-5.

20. Chen M, Yang J, Zhu L, Zhou C, Zhao H. Primary intrathoracic liposarcoma: a clinicopathologic study and prognostic analysis of 23 cases. J Cardiothorac Surg. 2014;9:119.

\section{Publisher's Note}

Springer Nature remains neutral with regard to jurisdictional claims in published maps and institutional affiliations. 